\title{
Primary Cilium by Polyinosinic:Polycytidylic Acid Regulates the Regenerative Migration of Beas-2B Bronchial Epithelial Cells
}

\author{
Bomi Gweon ${ }^{1}$, Tae-Kyu Jang ${ }^{2}$, Pham Xuan Thuy ${ }^{2}$ and Eun-Yi Moon ${ }^{2, *}$ \\ ${ }^{1}$ Department of Mechanical Engineering, Sejong University, Seoul 05006, \\ ${ }^{2}$ Department of Integrated Bioscience and Biotechnology, Sejong University, Seoul 05006, Republic of Korea
}

\begin{abstract}
The airway epithelium is equipped with the ability to resist respiratory disease development and airway damage, including the migration of airway epithelial cells and the activation of TLR3, which recognizes double-stranded (ds) RNA. Primary cilia on airway epithelial cells are involved in the cell cycle and cell differentiation and repair. In this study, we used Beas-2B human bronchial epithelial cells to investigate the effects of the TLR3 agonist polyinosinic:polycytidylic acid [Poly(I:C)] on airway cell migration and primary cilia (PC) formation. PC formation increased in cells incubated under serum deprivation. Migration was faster in Beas-2B cells pretreated with Poly(I:C) than in control cells, as judged by a wound healing assay, single-cell path tracking, and a Transwell migration assay. No changes in cell migration were observed when the cells were incubated in conditioned medium from Poly(l:C)-treated cells. PC formation was enhanced by Poly(l:C) treatment, but was reduced when the cells were exposed to the ciliogenesis inhibitor ciliobrevin A (CilioA). The inhibition of Beas-2B cell migration by CilioA was also assessed and a slight decrease in ciliogenesis was detected in SARS-CoV-2 spike protein (SP)-treated Beas-2B cells overexpressing ACE2 compared to control cells. Cell migration was decreased by SP but restored by Poly $(\mathrm{I}: \mathrm{C})$ treatment. Taken together, our results demonstrate that impaired migration by SP-treated cells can be attenuated by Poly(l:C) treatment, thus increasing airway cell migration through the regulation of ciliogenesis.
\end{abstract}

Key Words: Primary cilium, Poly(I:C), Airway epithelial cell, Beas-2B cell, Cell migration

\section{INTRODUCTION}

Airway epithelial cells not only provide physical resistance against pathogen invasion and allergens, they also protect against the development of respiratory diseases, such as asthma, through their activation of immune responses (Martin and Frevert, 2005; Holgate, 2008). However, the respiratory epithelium is frequently the site of injury, such as via the inhalation of toxic agents (Oertel et al., 2001). Airway epithelial cell migration plays a pivotal role in airway repair and remodeling (Wang et al., 2012), by adapting to changes in the local environment or repairing the epithelium after injury (Puchelle et al., 2006). Actin polymerization is involved in airway epithelial cell migration and repair (Zahm et al., 1991; de Bentzmann et al., 2000). Airway epithelial cell migration involves dedifferentiation, polarization, and redifferentiation and results in complete mucociliary regeneration (Castillon et al., 2002).

Toll-like receptors (TLRs) provide receptors for various pathogens, including viruses, which possess several components that activate TLR3, TLR4, and TLR7/8 (Koizumi et al., 2016). TLR agonists modulate wound regeneration in airway epithelial cells (Lewandowska-Polak et al., 2018). Among them, TLR3 plays an important role in the recognition of double-stranded (ds) RNA, triggering the production of a wide range of inflammatory mediators (Stowell et al., 2009) TLR3 also recognizes the synthetic ds RNA analog polyriboinosinic-polyribocytidylic acid [Poly(I:C)] (Jackson and Johnston, 2010). Poly(I:C) specifically induces the rapid secretion of chemokines and subsequently apoptosis, which may have detrimental effects on the damaged epithelium (Koizumi et al., 2016). In addition, TLR3 stimulates retinoic acid synthesis and signaling, thus promoting the healing of mammalian skin wounds (Kim and Garza, 2019) and damaged neonatal heart (Wang et al., 2018). However, whether Poly(I:C) contributes to the regeneration of bronchial epithelial damage through TLR3 activation is unknown.

\section{Open Access https://doi.org/10.4062/biomolther.2022.009}

This is an Open Access article distributed under the terms of the Creative Commons Attribution Non-Commercial License (http://creativecommons.org/licenses/by-nc/4.0/) which permits unrestricted non-commercial use, distribution, and reproduction in any medium, provided the original work is properly cited.
Received Jan 22, 2022 Revised Jan 29, 2022 Accepted Jan 30, 2022 Published Online Mar 1, 2022

\section{*Corresponding Author}

E-mail: eunyimoon@sejong.ac.kr

Tel: +82-2-3408-3768, Fax: +82-2-466-8768 
Primary cilia (PCs) are microtubule-based and antenna-like organelles that protrude from the plasma membrane surfaces of most vertebrate cells. PC plays a function as a transformer of intracellular signaling, including embryonic development and tissue homeostasis. Abnormalities in PCs are therefore major causes of developmental disorders and diseases in humans (Satir et al., 2010; Pedersen et al., 2012; Basten and Giles, 2013; Lee et al., 2019). Although PCs are not expressed in well-differentiated airway epithelial cells, they extend into the luminal layer of the epithelium during the cell cycle and cell differentiation and injury repair (Oertel et al., 2001). The protective effects of green tea components include an increase in the proportion of ciliated cells and upregulated ciliogenesis in airway cells (Kim et al., 2021). However, little is known about the relationship between cilium formation and cell migration as mediated by TLR3 activation.

In this study, the association between airway epithelium regeneration and cell migration induced by TLR3 activation and $\mathrm{PC}$ formation was investigated using Poly $(\mathrm{I}: \mathrm{C})$ and Beas$2 \mathrm{~B}$ human bronchial epithelial cells. In addition, the regenerative effect of Poly(l:C) on airway epithelial cell migration in response to wound healing was examined using the spike protein (SP) from severe acute respiratory syndrome coronavirus 2 (SARS-CoV-2), the virus responsible for the current pandemic (Wu et al., 2020). Our results demonstrate the ability of Poly $(\mathrm{I}: \mathrm{C})$ to stimulate regenerative cell migration after the loss of epithelial cell integrity.

\section{MATERIALS AND METHODS}

\section{Reagents}

Polyinosinic:polycytidylic acid [Poly(I:C)], MTT [3(4,5-dimethyl-thiazol-2-yl)-2,5-diphenyl tetrazolium bromide] and mouse antibodies which are reactive with acetylated tubulin (T7451) were from Sigma-Aldrich Co (St. Louis, MO, USA). Polyethylenimine (PEI) was purchased from Polysciences, Inc (Warrington, PA, USA). Rabbit antibodies which are reactive with Flag (14793) were from Cell Signaling Technology Inc (Danvers, MA, USA). Rabbit antibodies which are reactive with Arl13b (17711-1-AP) were from Proteintech Group Inc (Rosemont, IL, USA). Goat anti-rabbit-Alexa 568 (A-11011) was obtained from Invitrogen (Calsbad, CA, USA). Human pCMV3-ACE2-Flag plasmid (HG10108-CF) and SARS-CoV-2 (2019-nCoV) spike protein (40592-V08B-B) were purchased from Sino Biological Inc (Wayne, PA, USA). Fibronectin, bovine collagen type I and bovine serum albumin (BSA) were purchased from Advanced BioMatrix (Carlsbad, CA, USA) Except where indicated, all other materials are obtained from the Sigma Chemical Co.

\section{Cell culture}

Beas-2B human bronchial epithelial cells (ATCC \# CRL9609) were obtained from Korea research institute of bioscience and biotechnology (KRIBB) cell bank (Daejeon, Korea). Cells were adapted and cultured as monolayers in Dullecco's modified Eagle's medium (DMEM) with supplement of $10 \%$ fetal bovine serum (FBS) (GIBCO, Grand Island, NY, USA), $2 \mathrm{mM}$ L-glutamine, 100 units $/ \mathrm{mL}$ penicillin and streptomycin (GIBCO). Cells were incubated at $37^{\circ} \mathrm{C}$ in a humidified atmosphere of $5 \% \mathrm{CO}_{2}$ maintenance. For the induction of primary cilia, cells were incubated in serum-starved media without
FBS for 24-36 $\mathrm{h}$.

\section{Cell adaptation}

Beas-2B cells were originally maintained in the coated dishes with FBS-free BEBE containing BEBM $^{\mathrm{TM}}$ bronchial epithelial cell growth basal medium along with all the additives, BEGM ${ }^{\mathrm{TM}}$ kit (Lonza/Clonetics Co., Basel Switzerland). Due to that ACE2 was not detected in Beas-2B cells cultured with BEBE under our experimental condition (data not shown), cells were adapted by replacing BEBE to DMEM with $10 \%$ FBS in the following manner. Culture medium was sequentially exchanged by feeding the fresh BEBE containing $25 \%$, $50 \%, 75 \%$ and $100 \%$ DMEM supplemented with $10 \%$ FBS every $24 \mathrm{~h}$. After 5 days, cells were sub-cultured into uncoated dishes with DMEM containing $10 \%$ FBS. The cells were fully adapted by sub-culture up to 40 times after the start of adaptation. All experiments were performed with Beas-2B cells at $40^{\text {th }}-60^{\text {th }}$ passage.

\section{Preparation of coated culture dish}

Culture dishes were coated as follows. The coating solution was prepared by a mixture of $0.01 \mathrm{mg} / \mathrm{mL}$ fibronectin $0.03 \mathrm{mg} / \mathrm{mL}$ bovine collagen type I and $0.01 \mathrm{mg} / \mathrm{mL}$ BSA dissolved in BEBE. Culture dishes were treated with coating solution and agitated gently to coat the entire surface. Then, culture dishes were incubated in a $37^{\circ} \mathrm{C}$ incubator overnight and coating solution was removed. Coated dishes were washed with PBS and stored at room temperature under light-protected condition up to one month.

\section{Cytotoxicity assay}

Cell survival was quantified by using colorimetric assay with MTT to measure intracellular succinate dehydrogenase content or by using luminescence assay with CellTiter-Glo substrate to measure intracellular ATP content (Jang et al., 2020). For MTT assay, confluent cells were cultured with various concentrations of each reagent for $24 \mathrm{~h}$. Cells were then incubated with $50 \mu \mathrm{g} / \mathrm{ml}$ of MTT at $37^{\circ} \mathrm{C}$ for $2 \mathrm{~h}$. Formazan formed by MTT were dissolved in dimethylsulfoxide (DMSO). Optical density (OD) was read at $540 \mathrm{~nm}$. For CellTiter-Glo assay, cells were treated with CellTiter-Glo substrate (Promega, Madison, WI, USA). Luminescence was detected by using Glomax ${ }^{\circledR}$ luminometer (Promega).

\section{Wound healing assay}

Cell migration was measured as described previously, with minor modifications (Lee et al., 2020b). Briefly, when Beas-2B cells reached confluence in a $35-\mathrm{mm}$ culture dish (Corning, NY, USA), three wound lines in the form of a cross were made by scratching the cellular layer with a plastic pipette tip. Floating cells were then washed out, and fresh medium was added. Cells were then incubated at $37^{\circ} \mathrm{C}$ in a humidified atmosphere of $5 \% \mathrm{CO}_{2}$ maintenance. Narrowing of the wound was then monitored using a phase-contrast microscope beginning $6 \mathrm{~h}$ after the scratch. The size of the wound at each time point was then quantified using $\mathrm{NIH}$ image analysis software (Image $\mathrm{J}$, version 1.62), and compared with that in the initiation of cell migration.

\section{Single cell path tracking}

Beas-2B cell culture dishes were focused under live cell imaging light microscope. Cells were treated with Poly $(\mathrm{I}: \mathrm{C})$ and 
video image of live cells were automatically taken for $18 \mathrm{~h}$. Cells were tracked every $30 \mathrm{~min}$. Changes in single cell path were analyzed by tracking program, ImageJ plugin MTrackJ (Version 1.5.0) and represented with two-dimensional Rose diagram. Each cell path was overlayed on a polar grid with the normalization of start points to the origin. Movement track was presented in coordinates for each cell (Go et al., 2013).

\section{Transwell cell migration assay}

Boyden chamber assay experiments were conducted using a 24-well Transwell system (6.5mm Transwell (\#3422), Corning) with each well separated by a microporous $10 \mu \mathrm{m}$ thin transparent polycarbonate membrane ( $8 \mu \mathrm{m}$ pore size) into an upper ("insert") and a lower chamber ("well"). A volume of $100 \mu \mathrm{L}$ containing 40,000 cells were plated to each insert and $600 \mu \mathrm{L}$ medium was added to the wells. Cells in 'insert' were allowed to migrate for $24 \mathrm{~h}$. Then, cells were fixed and stained in a $20 \%$ methanol $/ 0.1 \%$ crystal violet solution for $15 \mathrm{~min}$ at room temperature, followed by washing 'insert' with water to remove redundant staining. Cells on upper part of 'insert' membrane were wiped out. Cells migrated onto lower part of 'insert' membrane were photographed, counted and presented as bar graph (Lee et al., 2020b).

\section{Detection of primary cilia}

For the detection of primary cilia in vitro, cells were maintained in serum-free culture medium for 24-72 h (Pugacheva et al., 2007; Ott and Lippincott-Schwartz, 2012; Lim and Tang, 2015; Choi et al., 2016; Lee et al., 2019). Briefly, Beas-2B cells were grown on coverslip and then incubated with serum-deprived DMEM for $36 \mathrm{~h}$. Cells were fixed with $4 \%$ paraformaldehyde for $10 \mathrm{~min}$, washed three times with cold PBS, and permeabilized with PBST (0.1\% (v/v) Triton X-100 in PBS) for 10 $\min$. Then, cells were washed three times, and incubated with polyclonal anti-Arl13b antibodies diluted $(1: 1,000)$ in staining buffer $(0.01 \%$ Triton $\mathrm{X}-100,1 \%$ BSA in PBS) for $1 \mathrm{~h}$ at room temperature. After washing three times with PBS, cells were incubated with goat anti-rabbit IgG-Alexa 568 diluted $(1: 1,000)$ in staining buffer for $1 \mathrm{~h}$ at room temperature. Nucleus was visualized by staining cells with DAPI. After washing with PBS, cells were mounted on glass slide. Primary cilia were observed and photographed at 1,000 $\times$ magnification under a fluorescence microscope (Nikon, Tokyo, Japan).

\section{Transfection of nucleic acids}

ACE2, receptor for SARS-Cov2 spike protein was overexpressed by the transfection of cells with pCMV3-ACE2-Flag plasmid DNA, which was accompanied with pCMV for control group, respectively. Each plasmid DNA was transfected into cells as follows (Lee et al., 2019). Briefly, each nucleic acid and Polyethylenimine (PEI) (Polysciences, Inc.) was diluted in serum-free medium and incubated for $5 \mathrm{~min}$, respectively. The diluted nucleic acid and PEI were mixed by inverting and incubated for $20 \mathrm{~min}$ to form complexes. Pre-formed complexes were added directly to the cells and cells were incubated for 24-48 $\mathrm{h}$ until use (Lee et al., 2019).

\section{Western blotting}

Cells were lysed in ice-cold RIPA buffer (Triton X-100) containing protease inhibitor $(2 \mu \mathrm{g} / \mathrm{mL}$ aprotinin, $1 \mu \mathrm{M}$ pepstatin, $1 \mu \mathrm{g} / \mathrm{mL}$ leupeptin, $1 \mathrm{mM}$ phenylmetylsufonyl fluoride (PMSF), $5 \mathrm{mM}$ sodium fluoride (NaF) and $1 \mathrm{mM}$ sodium orthovanadate (Na3VO4). The protein concentration of the sample was measured using SMART ${ }^{\mathrm{TM}} \mathrm{BCA}$ protein assay kit (Pierce 23228) from iNtRON Biotech. Inc (Seoul, Korea). Same amount of heat-denatured protein in sodium dodecyl sulfate (SDS) sample buffer was separated in sodium dodecyl sulfate polyacrylamide gel electrophoresis (SDS-PAGE), and then transferred to nitrocellulose membrane by using electro blotter. Equal amount of loaded sample on membrane was verified by ponceau $\mathrm{S}$ staining. The membrane was incubated with blocking solution ( $5 \%$ non-fat skim milk in Tris-buffered saline with Tween 20 (TBST)), and then followed by incubation with the specific primary antibodies. Horse radish peroxidase (HRP)- or infrared (IR) fluorescence dye-conjugated secondary antibody were used for target-specific primary antibody. Immuno-reactive target bands were visualized by the reaction with enhanced chemiluminescence (ECL-PS250) (Dongin LS,
A

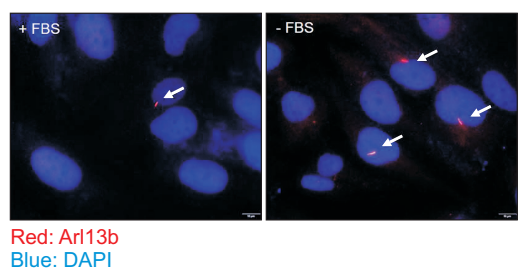

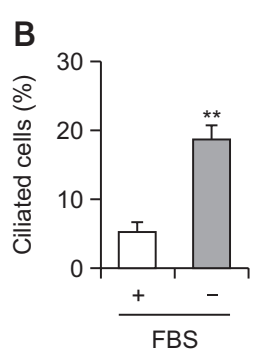

C

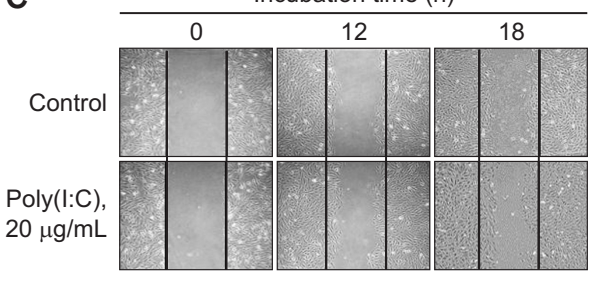

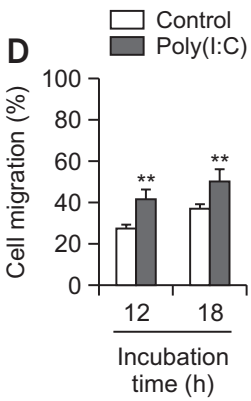

Fig. 1. Poly $(I: C)$ treatment enhanced Beas-2B cell migration with primary cilium formation. $(A, B) B$ Beas-2B cells were incubated in serumdeprived media without FBS for 24-36 h. The cells were fixed and stained with antibody against Arl13b (red) and DAPI (blue). The representative fluorescence image of primary cilia was shown. Processing such as changing brightness and contrast is applied equally to controls across the entire image (A). The ciliated cells incubated in the medium with (white) or without (grey) FBS were counted ( $n>500$ cells) (B). (C, D) Beas-2B cells were plated on $35-\mathrm{mm}^{2}$ dishes and incubated for $24 \mathrm{~h}$. A confluent monolayer of Beas-2B cells was then scratched with a sterile pipet tip, treated with $20 \mu \mathrm{g} / \mathrm{mL}$ Poly $(\mathrm{I}: \mathrm{C})$ and incubated for $12-18 \mathrm{~h}$. Migration of cells into the space left by the scratch was photographed using a phase-contrast microscope at 200× magnification (C). Percentage of cell migration was quantified by subtracting the empty area remained at each time point from that at the initiation using $\mathrm{NIH}$ image analysis software (version 1.62; National Institutes of Health), and compared to that of the 0-h time point. Percentage of cell migration was presented as bar graph (D). Data in bar graphs represented as means $\pm S D$. ${ }^{* *} p<0.01$, significantly different from control group with FBS (B) or Poly(I:C)-untreated group at each time point (D). 
Seoul, Korea) on X-ray film (Agfa HealthCare, Seoul, Korea) or by the detection of IRdye with Odyssey CLx Infrared Imaging System (LI-COR Biosciences, Lincoln, NE, Germany), respectively (Lee et al., 2019).

\section{Statistical analysis}

Experimental differences were verified for statistical significance using ANOVA and student's t-test. $p$-value of $<0.05$ and $<0.01$ was considered to be significant.

\section{RESULTS}

\section{Primary cilia and increased migration in Poly(l:C)-treated Beas-2B cells}

Because PCs are detected in the luminal layer of the epithelium (Oertel et al., 2001), we first examined the formation of PCs in Beas-2B human bronchial epithelial cells. As shown in Fig. $1 A$ and $1 B, P C$ formation was detected in Beas-2B cells incubated with complete medium containing $10 \%$ fetal bovine serum (+FBS). When the cells were incubated under serum deprivation (-FBS), PC formation increased. As bronchial epithelial cell migration plays a pivotal role in the airway repair and remodeling that occurs in respiratory diseases (Wang et al., 2012), the ability of Beas-2B cells to migrate in the setting of wound repair was determined. Wound formation was modeled by scratching a confluent cell monolayer. As Beas-2B cell migration was faster in serum-fed than in serum-starved cells (data not shown), +FBS medium was used in the cell migration experiment. After $12 \mathrm{~h}$ and $18 \mathrm{~h}$ incubation, roughly $29 \%$ and $38 \%$ of Beas-2B cells had migrated into the empty area (Fig. 1C, 1D). Previous studies have reported an effect of TLR3 and the TLR3 agonist Poly(I:C) on epithelial damage in the skin and heart (Nelson et al., 2015; Koizumi et al., 2016; Wang et al., 2018; Kim and Garza, 2019). We, therefore, examined the effects of Poly $(\mathrm{I}: \mathrm{C})$ on Beas-2B cell migration. No differences in Beas-2B cell viability were detected in response to the tested concentrations of Poly(l:C), as measured in a colorimetric assay using MTT and in a luminescence assay using the CellTiter-Glo substrate (data not shown). A Poly(I:C) concentration of $20 \mu \mathrm{g} / \mathrm{mL}$ significantly increased cell migration compared to the control, by about $45 \%$ and $30 \%$ at $12 \mathrm{~h}$
A

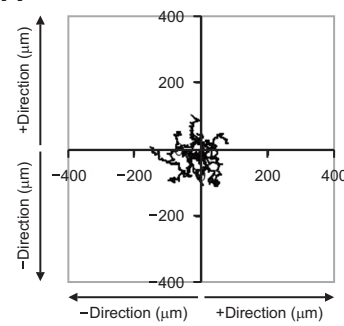

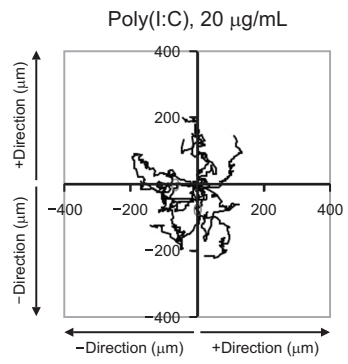

B

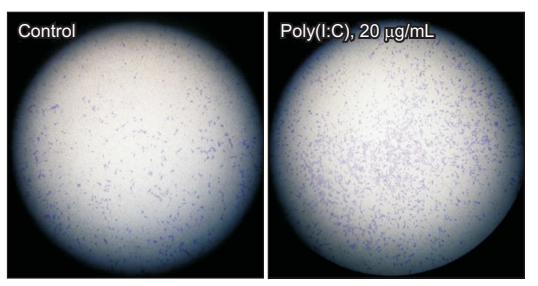

C

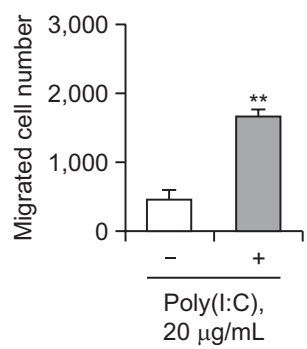

Fig. 2. Beas-2B cell migration by Poly $(I: C)$ treatment was confirmed by single cell path tracking and transwell migration assay. (A-C) Beas$2 \mathrm{~B}$ cells were treated with $20 \mu \mathrm{g} / \mathrm{mL}$ Poly(I:C). Changes in single cell path were analyzed by tracking program, ImageJ plugin MTrackJ (Version 1.5.0). Movement track was presented in coordinates for each cell on a polar grid with the normalization of start points to the origin (A). 40,000 cells and each conditioned media untreated (B, left) or treated (B, right) with Poly(l:C) were plated to each 'insert'. Cells were allowed to migrate for $24 \mathrm{~h}$. Then, cells were fixed, stained, and washed with water to remove redundant staining. Cells migrated onto lower part of 'insert' membrane were photographed (B), counted and presented as bar graph after cells on upper part of 'insert' membrane were wiped out. Data in bar graphs represented as means $\pm \mathrm{SD}$. ${ }^{* *} p<0.01$, significantly different from Poly(l:C)-untreated control group (C).

A

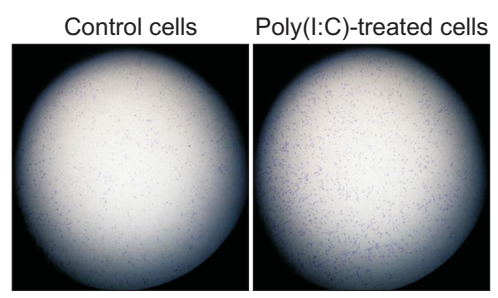

B

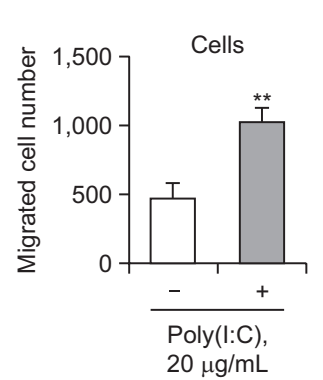

C Control conditioned medium (CM) Poly(I:C)-treated CM
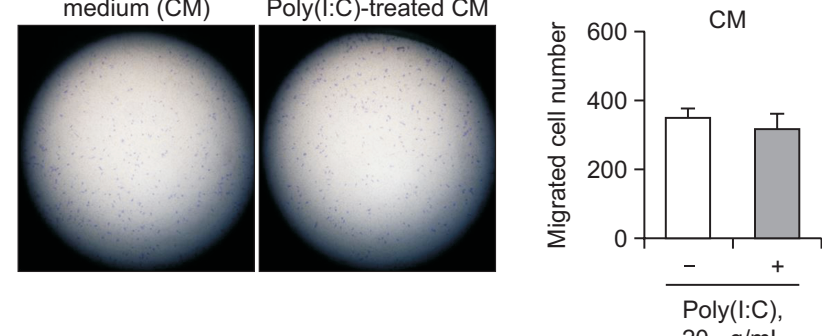

Fig. 3. Poly $(I: C)$-treated Beas-2B cell migration was more effective than control cell migration by Poly(l:C)-treated cell conditioned medium. (A-D) Beas-2B cells were treated with $20 \mu \mathrm{g} / \mathrm{mL}$ Poly(I:C). 40,000 cells untreated (A, left) or treated (A, right) with Poly(I:C) were plated to each 'insert' (A). 40,000 control Beas-2B cells in conditioned medium untreated (C, left) or treated (C, right) with Poly(I:C) were plated to each 'insert' $(C)$. Cells were allowed to migrate for $24 \mathrm{~h}$. Then, cells were fixed, stained, and washed with water to remove redundant staining. Cells migrated onto lower part of 'insert' membrane were photographed (A, C), counted and presented as bar graph after cells on upper part of 'insert' membrane were wiped out. Data in bar graphs represented as means \pm SD. ${ }^{* *} p<0.01$, significantly different from Poly(l:C)-untreated control group $(B)$. 
A

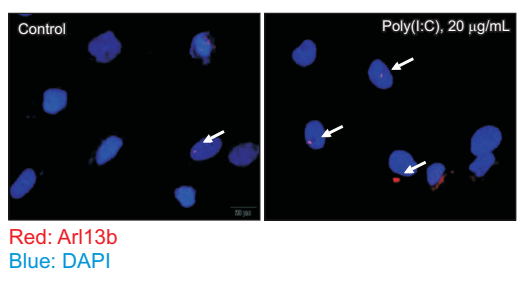

B

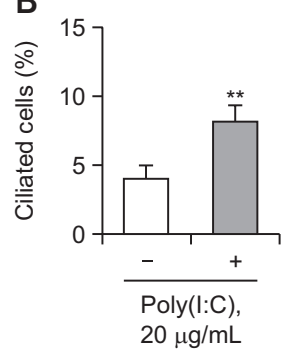

C

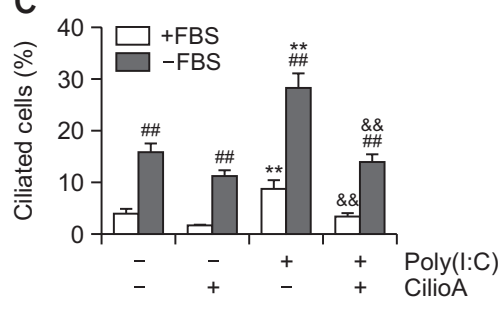

Fig. 4. Poly $(I: C)$ treatment increased primary cilium formation in Beas-2B cells. (A, B) Beas- $2 B$ cells were treated with $20 \mu \mathrm{g} / \mathrm{mL}$ Poly $(\mathrm{I}: \mathrm{C})$ and incubated in serum-deprived media without FBS for 24-36 h. The cells were fixed and stained with antibody against Arl13b (red) and DAPI (blue). The representative fluorescence image of primary cilia was shown. Processing (such as changing brightness and contrast is applied equally to controls across the entire image (A). The ciliated cells incubated in the medium with (white) or without (grey) FBS were counted ( $n>500$ cells) (B). (C) Beas-2B cells were treated with $20 \mu \mathrm{g} / \mathrm{ml}$ Poly(l:C) in the presence or absence of ciliogenesis inhibitor, ciliobrevin A (CilioA). Then, cells were incubated in serum-deprived media without FBS for 24-36 $\mathrm{h}$. The ciliated cells were stained with antibody against Arl13b and counted. Data in bar graphs represented as means \pm SD. ${ }^{* *} p<0.01$, significantly different from Poly(I:C)-untreated and CilioA-untreated group (B, C). ${ }^{\#} p<0.01$, significantly different from group incubated with FBS-containing medium at each condition (C). ${ }^{\& \&} p<0.01$, significantly different from Poly(I:C)-treated and CilioA-untreated group (C).

A

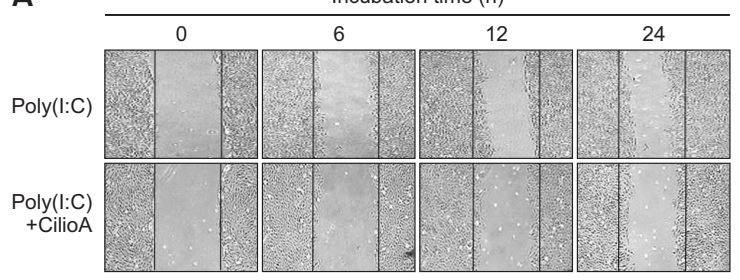

B

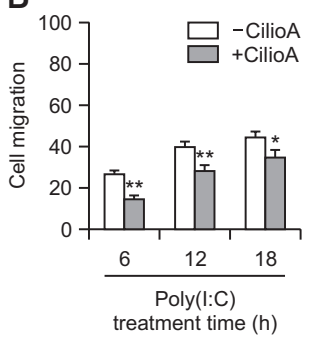

C Poly(I:C), $20 \mu \mathrm{g} / \mathrm{mL}$
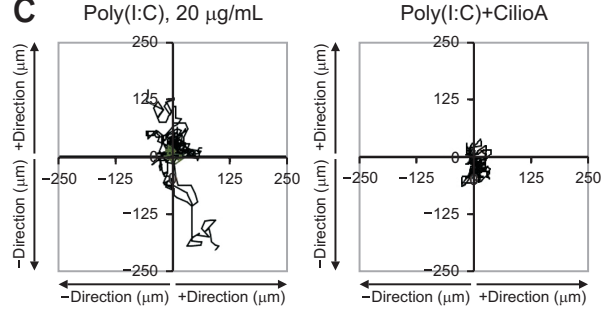

Fig. 5. Poly $(I: C)$-induced cell migration was inhibited by the incubation with ciliogenesis inhibitor. (A-C) Beas-2B cells were plated on $35-$ $\mathrm{mm}^{2}$ dishes and incubated for $24 \mathrm{~h}$. A confluent monolayer of Beas-2B cells was then scratched with a sterile pipet tip (A, B). Then, cells were treated with $20 \mu \mathrm{g} / \mathrm{mL}$ Poly $(\mathrm{I}: \mathrm{C})$ in the presence or absence of ciliogenesis inhibitor, ciliobrevin A (CilioA) and incubated for 6-24 h (AC). Migration of cells into the space left by the scratch was photographed using a phase-contrast microscope at $200 \times$ magnification (A). Percentage of cell migration was quantified by subtracting the empty area remained at each time point from that at the initiation using $\mathrm{NIH}$ image analysis software (version 1.62; National Institutes of Health), and compared to that of the 0-h time point. Percentage of cell migration was presented as bar graph. Data in bar graphs represented as means $\pm \mathrm{SD}$. ${ }^{*} p<0.05 ;{ }^{* *} p<0.01$, significantly different from CilioA-untreated group at each time point (B). Changes in single cell path were analyzed by tracking program, ImageJ plugin MTrackJ (Version 1.5.0). Movement track was presented in coordinates for each cell on a polar grid with the normalization of start points to the origin (C).

and $18 \mathrm{~h}$, respectively (Fig. 1C, 1D).

The effects of Poly $(\mathrm{I}: \mathrm{C})$ on Beas-2B cell migration were further studied using single-cell path tracking and a Transwell migration assay. The cells were treated with Poly $(\mathrm{I}: \mathrm{C})$ and the trajectories of the migrated cells were expressed in coordinates. The total coverage by migrating cells was larger in the Poly(I:C)-treated cells than in the control group (Fig. 2A). In a Transwell assay, Beas-2B cells were plated into the insert well and incubated with control or Poly(l:C)-containing medium for $24 \mathrm{~h}$. Cell migration by Poly(l:C)-treated cells was $\sim 3.2$-fold higher than that by control cells (Fig. 2B, 2C). These results suggest that $\mathrm{Poly}(\mathrm{I}: \mathrm{C})$ induces cell migration via $\mathrm{PC}$ formation.

\section{Poly(I:C)-induced Beas-2B cell migration is associated with cellular changes}

To examine the mechanism of cell migration in Poly(I:C)treated Beas-2B cells, the effects of conditioned medium from cells incubated with Poly $(I: C)$ and from control cells were tested. First, control and Poly(I:C)-treated Beas-2B cells were plated in the insert well of the Transwell and incubated with fresh complete medium for $24 \mathrm{~h}$. Cell migration of Poly(l:C)treated cells was $\sim 2.1$-fold higher than that of control cells (Fig. 3A, 3B). When the control cells were plated into the insert well of Transwell and incubated with conditioned medium from control or Poly(I:C)-treated cells, there were no differences in cell migration (Fig. 3C, 3D). This experiment showed that Poly $(\mathrm{I}: \mathrm{C})$ induced migration-related cellular changes rather than factors released into the medium.

\section{Poly(l:C)-induced Beas-2B cell migration depends on PC formation}

As the appearance of PC in airway epithelial cells is related to the cell cycle, and cell differentiation and injury repair (Oertel et al., 2001), we investigated the effects of Poly(I:C) on PC formation and in turn Poly(I:C)-induced Beas-2B cell migration. In cells treated with $20 \mu \mathrm{g}$ Poly $(\mathrm{I}: \mathrm{C}) / \mathrm{mL}$, PC formation was approximately two-fold higher than in control cells (Fig. 4A, 4B). To confirm the effects of Poly(I:C) on PC formation, Beas-2B cells were treated with ciliobrevin A (CilioA) to inhibit $\mathrm{PC}$ formation. When the cells were incubated with Poly(I:C), 
A

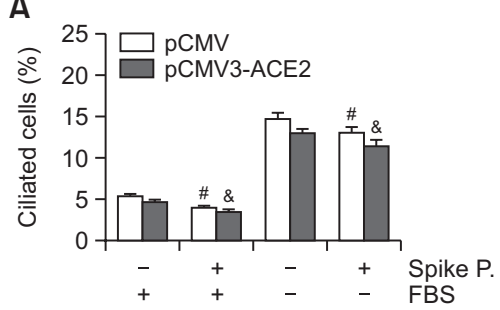

B

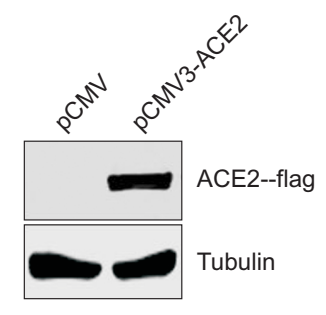

C

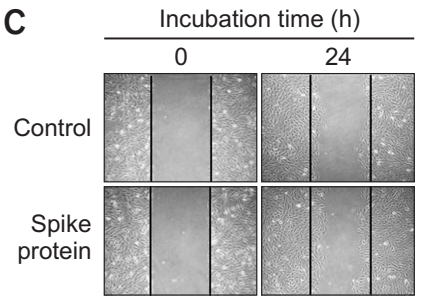

E

E

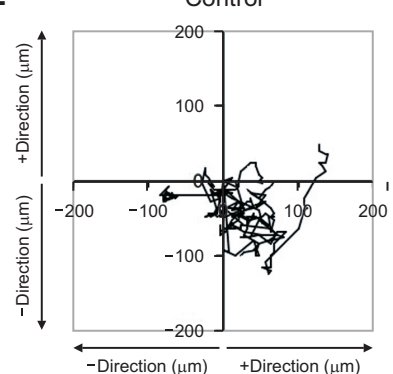

D

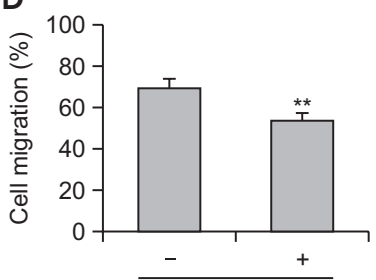

Spike protein $(25 \mathrm{ng} / \mathrm{mL})$

Spike protein

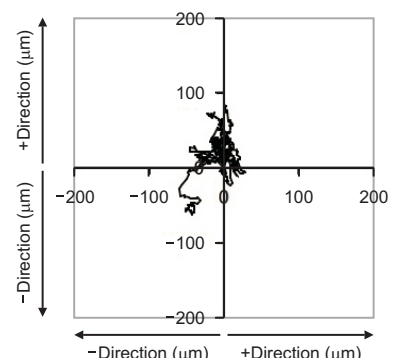

Fig. 6. Beas-2B cell migration was decreased by the treatment with SARS-CoV-2 (2019-nCoV) spike protein. (A, B) Beas-2B cells were transfected with pCMV control or pCMV3-ACE2-Flag plasmids DNA for $24 \mathrm{~h}$. Cells were treated with $25 \mathrm{ng} / \mathrm{mL}$ SARS-CoV-2 (2019-nCoV) spike protein in the presence or absence of FBS for another $24 \mathrm{~h}$. The cells were fixed and stained with antibody against Arl13b and DAPI. The ciliated cells were counted ( $n>500$ cells) (A). Cell lysates were prepared and the protein expression of ACE2-Flag was detected by western blotting using antibody against Flag (B). (C-E) Beas-2B cells were plated on 35- $\mathrm{mm}^{2}$ dishes and incubated for 24 h. A confluent monolayer of Beas-2B cells was then scratched with a sterile pipet tip. Then, cells were treated with $25 \mathrm{ng} / \mathrm{mL}$ spike protein and incubated for $24 \mathrm{~h}$. Migration of cells into the space left by the scratch was photographed using a phase-contrast microscope at 200× magnification (C). Percentage of cell migration was quantified by subtracting the empty area remained at each time point from that at the initiation using $\mathrm{NIH}$ image analysis software (version 1.62; National Institutes of Health), and compared to that of the 0-h time point. Percentage of cell migration was presented as bar graph (D). Changes in single cell path were analyzed by tracking program, ImageJ plugin MTrackJ (Version 1.5.0). Movement track was presented in coordinates for each cell on a polar grid with the normalization of start points to the origin (E). Data in bar graphs represented as means \pm SD. ${ }^{\#} p<0.05$, significantly different from pCMV-transfected and spike protein-untreated group incubated with or without FBS ${ }^{\&} p<0.05$, significantly different from pCMV3-ACE2-transfected and spike protein-untreated group incubated with or without FBS $(A, D) .{ }^{* *} p<0.01$, significantly different from spike protein-untreated group (D).

either in complete (+FBS) or serum-free (-FBS) medium, $\mathrm{PC}$ formation was significantly decreased when additionally treated with CilioA (Fig. 4C). The effects of PC formation on Poly(I:C)-induced Beas-2B cell migration were further examined in a wound healing assay and in single-cell path tracking. The results showed that Poly(l:C)-induced cell migration was reduced in response to CilioA treatment (Fig. 5A, 5B). After 6,12 , and $18 \mathrm{~h}$ incubation, $28 \%, 40 \%$, and $47 \%$ of the Poly(I:C)-treated Beas-2B cells had migrated into the empty area. These are $49 \%, 31 \%$, and $24 \%$ more cell migration at each time point, compared to the cells additionally treated with CilioA, respectively. Total coverage by the migrating cells was likewise less in the CilioA-treated group (Fig. 5C). These results suggest that $\mathrm{Poly}(\mathrm{I}: \mathrm{C})$-induced Beas-2B cell migration depends on PC formation.

\section{Poly $(\mathrm{I}: \mathrm{C})$ induces the regeneration of Beas-2B cells injured by SARS-CoV-2 spike protein}

Since SARS-CoV-2 infection depends on the cell surface receptor angiotensin-converting enzyme 2 (ACE2) (Hoffmann et al., 2020), which is abundantly expressed on respiratory cells (Lee et al., 2020a), we examined the effects of SARSCoV-2 SP on Beas-2B cell migration, including in cells treated with Poly(I:C). PC formation was inhibited by SP treatment. This inhibitory effect of SP on PC formation was further demonstrated by the transfection of ACE2 into Beas-2B cells us- ing the plasmid pCMV-ACE2 (Fig. 6A); ACE2 expression was confirmed by Western blotting (Fig. 6B). When Beas-2B cells were incubated with SP, cell migration after $24 \mathrm{~h}$ incubation was $52 \%$, compared to $71 \%$ in control cells (Fig. 6C, 6D). Similar results were obtained with single-cell path tracking, as total coverage was less in SP-treated than in control cells (Fig. $6 \mathrm{E})$. These data suggest that SP aggravates the regenerative migration of airway epithelial cells mediated by PC formation.

Then we examined whether Poly $(I: C)$ could counteract the inhibitory effect of SP on airway epithelial cell migration. Beas2B cells were treated with SP in the absence or presence of Poly(I:C). Under the latter condition, the SP-mediated inhibition of cell migration was ameliorated (Fig. 7A), as evidenced by $56 \%$ migration after $18 \mathrm{~h}$ in these cells but only $39 \%$ in the controls (Fig. 7B). Total coverage achieved by SP-mediated cell migration was larger in Poly(l:C)-post-treated cells than in Poly(I:C)-pretreated cells; in both cases coverage was greater than in the control group not treated with Poly(I:C) (Fig. 7C). According to these findings, Poly $(\mathrm{I}: \mathrm{C})$ induces Beas-2B cell injury induced by SARS-CoV-2 SP.

\section{DISCUSSION}

Airway epithelial cells play a role in the physical resistance to injury of the respiratory epithelium, such as induced by in- 
A

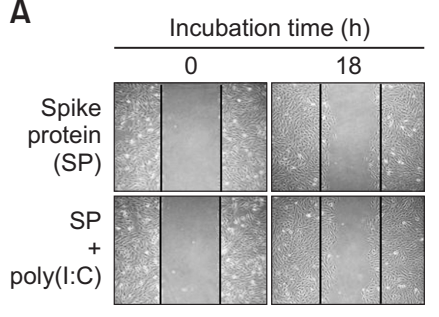

C

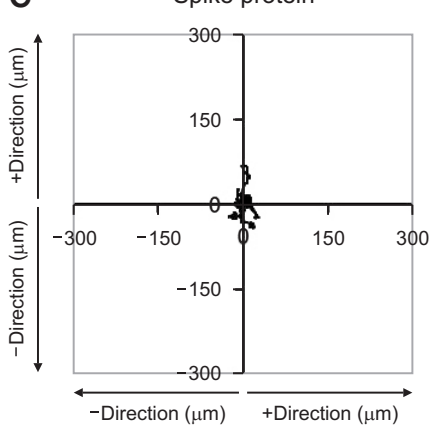

B

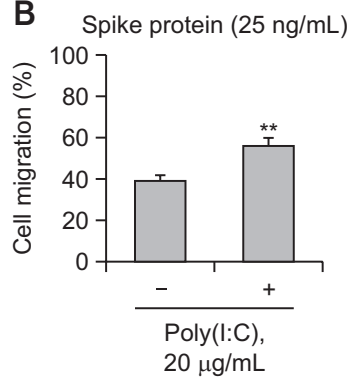

Poly(I:C)+Spike protein

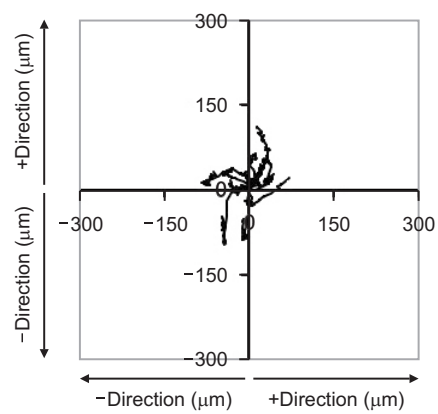

D

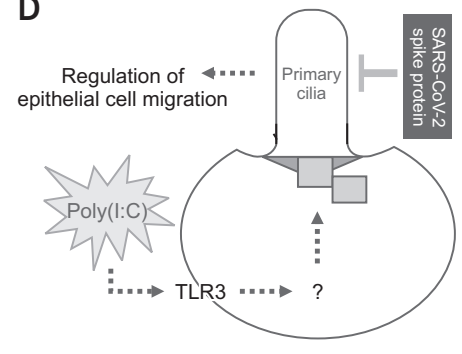

Spike protein+Poly(I:C)

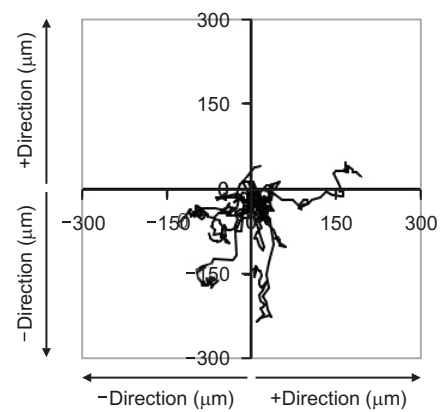

Fig. 7. Poly $(\mathrm{I}: \mathrm{C})$ treatment ameliorated the inhibitory Beas-2B cell migration by SARS-CoV-2 (2019-nCoV) spike protein. (A, B) Beas-2B cells were plated on $35-\mathrm{mm}^{2}$ dishes and incubated for $24 \mathrm{~h}$. A confluent monolayer of Beas-2B cells was then scratched with a sterile pipet tip. Then, cells were treated with $25 \mathrm{ng} / \mathrm{mL}$ spike protein in the presence or absence of $20 \mu \mathrm{g} / \mathrm{mL}$ Poly $(\mathrm{l}: \mathrm{C})$ and incubated for $18 \mathrm{~h}$. Migration of cells into the space left by the scratch was photographed using a phase-contrast microscope at 200× magnification (A). Percentage of cell migration was quantified by subtracting the empty area remained at each time point from that at the initiation using NIH image analysis software (version 1.62; National Institutes of Health), and compared to that of the 0-h time point. Percentage of cell migration was presented as bar graph. Data in bar graphs represented as means $\pm \mathrm{SD}$. ${ }^{* *} p<0.01$, significantly different from Poly $(\mathrm{I}: \mathrm{C})$-untreated group (B). (C) While cells were treated with $25 \mathrm{ng} / \mathrm{mL}$ spike protein, they were untreated (left), $12 \mathrm{~h}$ pre-treated (middle) or $12 \mathrm{~h}$ post-treated (right) with $20 \mu \mathrm{g} / \mathrm{mL}$ Poly(I:C), respectively for total $24 \mathrm{~h}$-incubation. Changes in single cell path were analyzed by tracking program, ImageJ plugin MTrackJ (Version 1.5.0). Movement track was presented in coordinates for each cell on a polar grid with the normalization of start points to the origin. (D) Scheme about action of Poly(I:C) on airway epithelial cell migration via PC formation. Airway epithelial cell migration is regulated by PC formation through some signaling factors (indicated as question mark) from the interaction of TLR3 and Poly(I:C) (black dotted lines). SARS-CoV-2 spike protein (SP) inhibits PC formation and airway epithelial cell migration (grey line). Our findings are presented by black dotted lines and grey inhibitory line.

haled toxic agents (Oertel et al., 2001) but also in protecting against the development of respiratory diseases (Martin and Frevert, 2005; Holgate, 2008), and in airway repair and remodeling (Wang et al., 2012), TLRs are receptors for many pathogens, and TLR agonists modulate wound regeneration in airway epithelial cells (Lewandowska-Polak et al., 2018). Among them, TLR3 participates in the response to ds RNA, including the synthetic analog Poly(I:C) (Jackson and Johnston, 2010). PCs extend into the epithelial lumen and are involved in the response of airway epithelial cells to injury (Oertel et al., 2001; Kim et al., 2021), but little is known about the relationship between PC formation and cell migration through TLR3 activation. In this study, we investigated the effects of Poly(I:C) on cell migration and $\mathrm{PC}$ formation leading to the regeneration of bronchial epithelial following damage by viral factors such as SARS-CoV-2 SP (Wu et al., 2020). We found that Poly(I:C) contributes to regenerative cell migration after the loss of epithelial cell integrity.

While many studies have examined cell migration in response to the TLR3 agonist Poly(I:C), the effects of ciliogenesis and Poly(I:C)-mediated TLR3 activation on the regenerative migration of airway epithelial cells remain poorly understood (Oertel et al., 2001; Wang et al., 2012; Nelson et al., 2015; Koizumi et al., 2016; Kim et al., 2021). Ciliogenesis occurs in well-differentiated airway epithelial cells, where it functions to protect the cells from injury and to repair damaged epithelium (Oertel et al., 2001; Kim et al., 2021). While Poly I:C has detrimental effects on injured epithelial cells (Koizumi et al., 2016), TLR3 stimulation correlates with the regeneration of injured skin or heart tissue (Nelson et al., 2015; Wang et al., 2018). By investigating Poly(I:C)-indued TLR3 activation, our study provides insights into the therapeutic relationship between PC formation and regenerative migration in airway cells.

Cilia are assembled during the G0/G1 phase, disassembled during the $\mathrm{S}$ phase, and are not detectable during the G2/M phase of the cell cycle. PCs are observed during the G1 phase (Avasthi and Marshall, 2012). Because serum starvation induces cell cycle arrest at G (Huang et al., 2018), the in vitro incubation of Beas-2B cells under serum deprivation will induce PC formation (Fig. 1). Our data indicate that the Poly(I:C)-mediated increase in airway epithelial cell migration is associated with an increase in ciliogenesis under conditions detrimental to cells, such as serum deprivation (Fig. 1, 2).

Previous studies have reported an association between several different molecules and signaling pathways in TLR3 
activation. TLR3 mediates repair and regeneration through glycolysis-dependent YAP1-regulation (Wang et al., 2018). Activation of the TLR3/PI3K/Akt pathway by Poly(I:C) prevents myocardial ischemia/reperfusion injury (Chen et al., 2020), and the treatment of ischemic brain tissues with Poly(I:C) increases Hsp27, Hsp70, and $\mathrm{Bcl} 2$ levels while reducing the Bax level (Wang et al., 2014). We found an increase in Beas$2 \mathrm{~B}$ cell migration by Poly(I:C), mediated by cellular changes, whereas Beas-2B cell migration was not facilitated by the conditioned medium of Poly(I:C)-treated cells (Fig. 3). However, the signaling molecules that regulate $\mathrm{PC}$ formation and cell migration in response to Poly $(\mathrm{I}: \mathrm{C})$ are thus far unknown, as is their mechanism of action.

As PC formation plays a role in restoring the damaged airway epithelium (Oertel et al., 2001; Kim et al., 2021), we investigated Poly(I:C)-mediated cell migration and specifically $\mathrm{PC}$ formation using the ciliogenesis inhibitor, CilioA. Poly(I:C)induced ciliogenesis was reduced by co-incubation of Beas2B cells with CilioA (Fig. 4). We also found that CilioA inhibits Poly(l:C)-mediated cell migration, evidenced in a wound healing assay and by single-cell path tracking (Fig. 5). These results suggest that the mechanism linking airway cell migration and the response to Poly (I:C) involves PC formation, and therefore the potential of $\mathrm{PC}$ as a novel therapeutic target to increase the regenerative migration of airway epithelial cells.

To better understand the role of Poly(l:C)-mediated cell migration in the regeneration of a damaged epithelium, we examined the effects of SARS-CoV-2 SP on PC formation and airway epithelial cell migration. A slight decrease in $P C$ formation was observed in SP-treated cells compared to control cells. The abnormal detection of extracellular signals by SP-exposed cilia was suggested by the impaired PC formation in cells overexpressing ACE2, the SP receptor on airway epithelial cells (Fig. 6). SP also decreased airway epithelial cell migration, both in a wound healing assay and in a singlecell tracking assay, whereas migration recovered in response to Poly(I:C) treatment (Fig. 7). According to these results, Poly(I:C) can prevent or attenuate the airway epithelial changes induced by environmental factors.

While neither the signaling molecules that regulate PC formation and cell migration nor the mechanism linking PC formation to Poly(I:C)-mediated cell migration is known, our results indicate that Poly $(\mathrm{I}: \mathrm{C})$ stimulates regenerative epithelial cell migration and PC formation after wound formation. Our findings support a pathway in which airway epithelial cell migration is regulated by PC formation, via as-yet-unidentified signaling factors that promote the interaction between $\mathrm{PC}$ and TLR3 (Fig. 7D). Poly(I:C) may therefore be a therapeutic molecule able to induce the repair of airway epithelial cells damaged by SARS-CoV-2 that can ameliorate the structural changes in the airway caused by exposure to other injurious agents.

\section{CONFLICT OF INTEREST}

The authors declare no competing financial and non- financial interests.

\section{ACKNOWLEDGMENTS}

We sincerely thank So-Jeong Park and Seo-Yeon Choi for their technical assistance to experiments of Transwell migration assay and single cell path tracking. This research was supported by the Basic Research Program through the National Research Foundation of Korea (NRF) funded by the Ministry of Science and ICT (grant number 2021R1A4A5033289).

\section{REFERENCES}

Avasthi, P. and Marshall, W. F. (2012) Stages of ciliogenesis and regulation of ciliary length. Differentiation 83, S30-S42.

Basten, S. G. and Giles, R. H. (2013) Functional aspects of primary cilia in signaling, cell cycle and tumorigenesis. Cilia 2, 6.

Castillon, N., Hinnrasky, J., Zahm, J. M., Kaplan, H., Bonnet, N., Corlieu, P., Klossek, J. M., Taouil, K., Avril-Delplanque, A., Peault, B. and Puchelle, E. (2002) Polarized expression of cystic fibrosis transmembrane conductance regulator and associated epithelial proteins during the regeneration of human airway surface epithelium in three-dimensional culture. Lab. Invest. 82, 989-998.

Chen, E., Chen, C., Niu, Z., Gan, L., Wang, Q., Li, M., Cai, X., Gao, R., Katakam, S., Chen, H., Zhang, S., Zhou, R., Cheng, X., Qiu, Y., Yu, H., Zhu, T. and Liu, J. (2020) Poly(I:C) preconditioning protects the heart against myocardial ischemia/reperfusion injury through TLR3/PI3K/Akt-dependent pathway. Signal Transduct. Target. Ther. 5, 216.

Choi, H., Shin, J. H., Kim, E. S., Park, S. J., Bae, I. H., Jo, Y. K., Jeong, I. Y., Kim, H. J., Lee, Y., Park, H. C., Jeon, H. B., Kim, K. W., Lee, T. R. and Cho, D. H. (2016) Primary cilia negatively regulate melanogenesis in melanocytes and pigmentation in a human skin model. PLoS ONE 11, e0168025.

de Bentzmann, S., Polette, M., Zahm, J. M., Hinnrasky, J., Kileztky, C., Bajolet, O., Klossek, J. M., Filloux, A., Lazdunski, A. and Puchelle, E. (2000) Pseudomonas aeruginosa virulence factors delay airway epithelial wound repair by altering the actin cytoskeleton and inducing overactivation of epithelial matrix metalloproteinase-2. Lab. Invest. 80, 209-219.

Go, A., Ryu, Y. K., Lee, J. W. and Moon, E. Y. (2013) Cell motility is decreased in macrophages activated by cancer cell-conditioned medium. Biomol. Ther. (Seoul) 21, 481-486.

Hoffmann, M., Kleine-Weber, H., Schroeder, S., Kruger, N., Herrler, T., Erichsen, S., Schiergens, T. S., Herrler, G., Wu, N. H., Nitsche, A., Muller, M. A., Drosten, C. and Pohlmann, S. (2020) SARS-CoV-2 cell entry depends on ACE2 and TMPRSS2 and is blocked by a clinically proven protease inhibitor. Cell 181, 271-280.e8.

Holgate, S. T. (2008) The airway epithelium is central to the pathogenesis of asthma. Allergol. Int. 57, 1-10.

Huang, Y., Fu, Z., Dong, W., Zhang, Z., Mu, J. and Zhang, J. (2018) Serum starvation-induces down-regulation of Bcl-2/Bax confers apoptosis in tongue coating-related cells in vitro. Mol. Med. Rep. 17, 5057-5064.

Jackson, D. J. and Johnston, S. L. (2010) The role of viruses in acute exacerbations of asthma. J Allergy Clin. Immunol. 125, 1178-1187.

Jang, J. W., Lee, J. W., Yoon, Y. D., Kang, J. S. and Moon, E. Y. (2020) Bisphenol $A$ and its substitutes regulate human B cell survival via Nrf2 expression. Environ. Pollut. 259, 113907.

Kim, D. and Garza, L. A. (2019) Hypothesis: wound-induced TLR3 activation stimulates endogenous retinoic acid synthesis and signalling during regeneration. Exp. Dermatol. 28, 450-452.

Kim, J., Choi, H., Choi, D. H., Park, K., Kim, H. J. and Park, M. (2021) Application of green tea catechins, polysaccharides, and flavonol prevent fine dust induced bronchial damage by modulating inflammation and airway cilia. Sci. Rep. 11, 2232.

Koizumi, Y., Nagase, H., Nakajima, T., Kawamura, M. and Ohta, K. (2016) Toll-like receptor 3 ligand specifically induced bronchial epithelial cell death in caspase dependent manner and functionally upregulated Fas expression. Allergol. Int. 65 Suppl, S30-S37.

Lee, I. T., Nakayama, T., Wu, C. T., Goltsev, Y., Jiang, S., Gall, P. A., 
Liao, C. K., Shih, L. C., Schurch, C. M., Mcllwain, D. R., Chu, P., Borchard, N. A., Zarabanda, D., Dholakia, S. S., Yang, A., Kim, D., Chen, H., Kanie, T., Lin, C. D., Tsai, M. H., Phillips, K. M., Kim, R., Overdevest, J. B., Tyler, M. A., Yan, C. H., Lin, C. F., Lin, Y. T., Bau, D. T., Tsay, G. J., Patel, Z. M., Tsou, Y. A., Tzankov, A., Matter, M. S., Tai, C. J., Yeh, T. H., Hwang, P. H., Nolan, G. P., Nayak, J. V. and Jackson, P. K. (2020a) ACE2 localizes to the respiratory cilia and is not increased by ACE inhibitors or ARBs. Nat. Commun. 11, 5453.

Lee, J., Yoon, S. S., Thuy, P. X. and Moon, E. Y. (2020b) Synovial cell migration is associated with $\mathrm{B}$ cell activating factor expression increased by TNFalpha or decreased by KR33426. Biomol. Ther. (Seoul) 28, 405-413.

Lee, J. W., Kim, H. S. and Moon, E. Y. (2019) Thymosin beta-4 is a novel regulator for primary cilium formation by nephronophthisis 3 in HeLa human cervical cancer cells. Sci. Rep. 9, 6849.

Lewandowska-Polak, A., Brauncajs, M., Jarzebska, M., Pawelczyk, M., Kurowski, M., Chalubinski, M., Makowska, J. and Kowalski, M. L. (2018) Toll-like receptor agonists modulate wound regeneration in airway epithelial cells. Int. J. Mol. Sci. 19, 2456.

Lim, Y. S. and Tang, B. L. (2015) A role for Rab23 in the trafficking of Kif17 to the primary cilium. J. Cell Sci. 128, 2996-3008.

Martin, T. R. and Frevert, C. W. (2005) Innate immunity in the lungs. Proc. Am. Thorac. Soc. 2, 403-411.

Nelson, A. M., Reddy, S. K., Ratliff, T. S., Hossain, M. Z., Katseff, A. S., Zhu, A. S., Chang, E., Resnik, S. R., Page, C., Kim, D., Whittam, A. J., Miller, L. S. and Garza, L. A. (2015) dsRNA released by tissue damage activates TLR3 to drive skin regeneration. Cell Stem Cell $17,139-151$.

Oertel, M., Graness, A., Thim, L., Buhling, F., Kalbacher, H. and Hoffmann, W. (2001) Trefoil factor family-peptides promote migration of human bronchial epithelial cells: synergistic effect with epidermal growth factor. Am. J. Respir. Cell Mol. Biol. 25, 418-424.

Ott, C. and Lippincott-Schwartz, J. (2012) Visualization of live primary cilia dynamics using fluorescence microscopy. Curr. Protoc. Cell Biol. Chapter 4, Unit 4.26.

Pedersen, L. B., Schroder, J. M., Satir, P. and Christensen, S. T. (2012)
The ciliary cytoskeleton. Compr. Physiol. 2, 779-803.

Puchelle, E., Zahm, J. M., Tournier, J. M. and Coraux, C. (2006) Airway epithelial repair, regeneration, and remodeling after injury in chronic obstructive pulmonary disease. Proc. Am. Thorac. Soc. 3 , 726-733.

Pugacheva, E. N., Jablonski, S. A., Hartman, T. R., Henske, E. P. and Golemis, E. A. (2007) HEF1-dependent Aurora A activation induces disassembly of the primary cilium. Cell 129, 1351-1363.

Satir, P., Pedersen, L. B. and Christensen, S. T. (2010) The primary cilium at a glance. J. Cell Sci. 123, 499-503.

Stowell, N. C., Seideman, J., Raymond, H. A., Smalley, K. A., Lamb, R. J., Egenolf, D. D., Bugelski, P. J., Murray, L. A., Marsters, P. A., Bunting, R. A., Flavell, R. A., Alexopoulou, L., San Mateo, L. R., Griswold, D. E., Sarisky, R. T., Mbow, M. L. and Das, A. M. (2009) Long-term activation of TLR3 by poly(I:C) induces inflammation and impairs lung function in mice. Respir. Res. 10, 43.

Wang, P. F., Fang, H., Chen, J., Lin, S., Liu, Y., Xiong, X. Y., Wang, Y. C., Xiong, R. P., Lv, F. L., Wang, J. and Yang, Q. W. (2014) Polyinosinic-polycytidylic acid has therapeutic effects against cerebral ischemia/reperfusion injury through the downregulation of TLR4 signaling via TLR3. J. Immunol. 192, 4783-4794.

Wang, W. C., Kuo, C. Y., Tzang, B. S., Chen, H. M. and Kao, S. H. (2012) IL-6 augmented motility of airway epithelial cell BEAS-2B via Akt/GSK-3beta signaling pathway. J. Cell. Biochem. 113, 35673575.

Wang, X., Ha, T., Liu, L., Hu, Y., Kao, R., Kalbfleisch, J., Williams, D. and Li, C. (2018) TLR3 mediates repair and regeneration of damaged neonatal heart through glycolysis dependent YAP1 regulated miR-152 expression. Cell Death Differ. 25, 966-982.

Wu, F., Zhao, S., Yu, B., Chen, Y. M., Wang, W., Song, Z. G., Hu, Y., Tao, Z. W., Tian, J. H., Pei, Y. Y., Yuan, M. L., Zhang, Y. L., Dai, F. H., Liu, Y., Wang, Q. M., Zheng, J. J., Xu, L., Holmes, E. C. and Zhang, Y. Z. (2020) A new coronavirus associated with human respiratory disease in China. Nature 579, 265-269.

Zahm, J. M., Chevillard, M. and Puchelle, E. (1991) Wound repair of human surface respiratory epithelium. Am. J. Respir. Cell Mol. Biol. 5, 242-248. 